Since $q<3 n^{-1 / 4}$, the expression in the parenthesis is positive for $n$ large enough, and $\sum_{\mu=1}^{k}\left|f\left(e^{i \theta_{\mu}}\right)\right|<2^{n}$, unless $q=0$. But $q=0$ means that every $z_{\nu}=-1$, and $f(z)=(z+1)^{n}$.

The theorem has thus been proved for large values of $n$.

(5) For small values of $n$ the theorem is incorrect. For example, if $f(z)=(z+1) \cdot(z-1 / 2)$, then $\left|f\left(e^{i \theta}\right)\right|$ has relative extrema for $\theta=0, \pi$, arc $\cos (1 / 8)$, and the sum of the values of $\left|f\left(e^{i \theta}\right)\right|$ at these places is $4.18>2^{2}$.

$f(z)=(z+1)^{2} \cdot(z-1 / 3)$ is another example in which $\sum\left|f\left(z_{\mu}\right)\right|$ $=8.14>2^{3}$.

Amherst College

\title{
ON LOCALLY SIMPLE CURVES
}

\section{G. T. WHYBURN}

1. Introduction. A continuous transformation $f(A)=B$ is locally simple provided that for each $x \in A$ there is a neighborhood $U$ of $x$ such that $U \cdot f^{-1}(y)$ contains at most one point for each $y \in B$. For the case where $A$ is a circle and $B$ is planar, such mappings have been studied recently by Morse and Heins [1] ${ }^{1}$ and used effectively in investigating meromorphic and other functions by topological methods.

In this paper topological characterizations will be obtained for those continua (compact connected metric spaces) which admit locally simple representations on the circle, that is, which are the image of the circle under some locally simple mapping.

It is clear that if $A$ is compact and metric, a mapping (continuous) $f(A)=B$ is locally simple if and only if there exists an $e>0$ such that any subset of $A$ of diameter not greater than $e$ maps topologically onto its image under $f$ or, equivalently, for any $y \in B$, any two distinct points of $f^{-1}(y)$ are at a distance greater than $e$ apart.

A simple arc $a b$ in a continuum $M$ will be called doubly extensible in $M$ provided $M$ contains a simple arc $a_{1} a b b_{1}$ such that every point of $a b$ is an interior point of $a_{1} a b b_{1}$. A continuum homeomorphic with the letter $\theta$ or with the figure 8 is called a $\theta$-curve or an 8-curve respectively; one homeomorphic with the sum of two disjoint circles plus a segment joining them with just an end point in each will be called a

Presented to the Society, April 26, 1947; received by the editors March 13, 1947.

1 Numbers in brackets refer to the bibliography at the end of the paper. 
dumbbell curve; and one homeomorphic with a circumference plus a radius will be called a lariat curve. A set is cyclic if it is connected and has no cut point.

\section{Imbedding results.}

(2.1) Theorem. In a locally connected continuum $M$ any doubly extensible arc $a b$ is contained in either a (a) simple closed curve, (b) $\theta$-curve, (c) 8-curve, (d) dumbbell curve, (e) lariat curve, or (f) maximal arc in $M$, where all end points of curves (e) or (f) are end points of $M$.

Proof. Let $a_{1} a b b_{1}$ be an arc in $M$ containing $a b$ in its interior. If $a$ is not a cut point of $M$, let $u v$ be an arc in $M-a$ such that $u \in a_{1} a$, $v \in a b_{1}$ and $u v \cdot a_{1} b_{1}=u+v$. Let $C_{a}=u v+\operatorname{arc} u a v$ of $a_{1} b_{1}$. If $v \in b b_{1}, C_{a}$ is a simple closed curve containing $a b$. Hence we may suppose $v \in a b$ $-a-b$. Similarly if $b$ is not a cut point, we construct an arc $r s$ in $M-b$ such that $s \in b b_{1}, r \in a b-a-b$ and let $C_{b}=r s+\operatorname{arc} r b s$ of $a_{1} b_{1}$.

If $a$ is a cut point of $M$, let $R$ be a component of $M-a$ not containing $a b-a$. If $R+a$ contains a simple closed curve $N_{a}$, let $C_{a}$ be $N_{a}+$ an $\operatorname{arc} a q$ in $R+a$, where $a q \cdot N_{a}=q$, and $a q=a$ if $a \in C_{a}$. Otherwise let $C_{a}$ be an arc $a e$ in $R+a$ joining $a$ and an end point $e$ of $M$. Construct $C_{b}$ similarly if $b$ is a cut point.

Now if neither $a$ nor $b$ is a cut point, the open $\operatorname{arcs} u v-u-v$ and $r s-r-s$ may intersect. In this case their sum $+u+s$ contains an arc $u s$ intersecting $a_{1} b_{1}$ in just $u+s$, and clearly $u s+u a b s$ (of $a_{1} b_{1}$ ) is a simple closed curve containing $a b$. If these arcs are disjoint, let $C=C_{a}+C_{b}+a b$. Then $C$ contains $a b$ and is a $\theta$-curve if $r$ precedes $v$ on $a b$, an 8-curve if $v=r$, and a dumbbell curve if $r$ follows $v$ on $a b$.

In case at least one of the points $a$ and $b$ is a cut point, the sets $C_{a}$ and $C_{b}$ are disjoint and the set $C=C_{a}+C_{b}+a b$ is a dumbbell curve, a simple arc, or a lariat curve and all end points of $C$ are end points of $M$.

(2.11) CoRollary. If $M$ has no end points, any doubly extensible arc in $M$ is contained in a curve of one of the types (a)-(d) in $M$.

(2.2) Theorem. Every doubly extensible arc ab in a cyclic locally connected continuum $C$ is contained in a cyclic graph in $C$.

For let $a_{1} a b b_{1}$ be an arc in $C$ containing $a b$ in its interior. Let $S$ be the set of all $x \in a b$ such that there exists a cyclic graph $G_{x}$ in $C$ intersecting $a b_{1}$ in an arc $a y$ where $y \in x b_{1}-x$. Then $S$ is nonempty since $M-a$ contains an arc wz intersecting $a_{1} b_{1}$ in just $w$ and $z$ and so that $w \in a_{1} a, z \in a b_{1}$. Also $S$ is open in $a b$ because if $x \in S$ and $G_{x}$ and $a y$ are determined by $x$, clearly they serve also for any $x^{\prime} \in a y-y$. But $S$ 
is also closed in $a b$. For let $p$ be a limit point of $S$ and suppose $p$ not in $S$. Let $u v$ be an arc in $C-p$ with $u \in a p, v \in p b_{1}$ and $u v \cdot a b_{1}=u+v$. Then there is a point $x \in S \cdot(u p-u)$. Let $G_{x}$ be the corresponding cyclic graph with $G_{x} \cdot a b_{1}=a y$. Then $y \in u p$, as otherwise $p \in S$. But then if $q$ is the last point of $G_{x}$ on $u v, G_{x}+y p v+q v$ is a cyclic graph in $C$ intersecting $a b_{1}$ in just $a v$ and $v \in p b_{1}-p$.

Thus $S$ is both open and closed in $a b$ and hence $S=a b$. Accordingly there exists a cyclic graph $G_{b}$ intersecting $a b_{1}$ in $a y$, where $y \in b b_{1}-b$, and hence containing $a b$.

Since every arc in a cyclic graph, or in any graph without end points, obviously is doubly extensible in that graph, we have

(2.21) Corollary. In a cyclic locally connected continuum $C, a$ simple arc is doubly extensible if and only if it is imbeddable in a cyclic graph in $C$.

A simple example of a cyclic locally connected continuum containing a non-extensible arc is afforded by the right triangle with vertices $(0,0),(1,0)$, and $(1,1)$ together with an infinite sequence of vertical segments joining the hypotenuse and base and converging to the origin. Then any simple arc in this continuum containing infinitely many of the vertical segments fails to be doubly extensible.

3. Locally simple representations on the circle. We begin with an addition theorem.

(3.1) THEOREM. If the continua $C_{1}$ and $C_{2}$ have locally simple representations on the circle and $C_{1} \cdot C_{2} \neq 0$, then $C_{1}+C_{2}$ has a locally simple representation on the circle.

Proof. Let $p \in C_{1}-C_{1} \cdot C_{2}, q \in C_{2}-C_{1} \cdot C_{2}$, and let $f_{1}(z)$ and $f_{2}(z)$ be locally simple mappings of the circle $|z|=1$ onto $C_{1}$ and $C_{2}$ respectively so chosen that $f_{1}(1)=p, f_{2}(1)=q$. Let $\theta_{1}$ be the least value of $\theta$ in the range $\pi / 2<\theta<\pi$ such that $f_{1}\left(e^{i 4 \theta}\right)=w \in C_{2}$ and let $\theta_{2}$ be the least value of $\theta$ in the range $3 \pi / 2<\theta<2 \pi$ such that $f_{2}\left(e^{i 4 \theta}\right)=s \in C_{1}$. Let $\alpha$ and $\beta$ be positive or 0 angles less than $\pi / 2$ such that

$$
f_{2}\left[e^{4 i\left(\theta_{1}-\alpha\right)}\right]=w, \quad f_{1}\left[e^{4 i\left(\theta_{2}-\beta\right)}\right]=s .
$$

Now let us define $f(z)$ for $z=e^{i \theta}, 0 \leqq \theta \leqq 2 \pi$, as follows:

$$
\begin{array}{rlr}
f(z) & =f_{1}\left(e^{i 4 \theta}\right) & \quad\left(\text { for } 0 \leqq \theta \leqq \theta_{1}\right) \\
& =f_{2}\left(e^{i 4 \phi}\right) \quad\left(\text { for } \theta_{1} \leqq \theta \leqq \theta_{2}, \text { where } \phi=\theta-\alpha\left(\theta_{2}-\theta\right) /\left(\theta_{2}-\theta_{1}\right)\right) \\
& =f_{1}\left(e^{i 4 \psi}\right) \quad\left(\text { for } \theta_{2} \leqq \theta \leqq 2 \pi, \text { where } \psi=\theta-\beta(2 \pi-\theta) /\left(2 \pi-\theta_{2}\right)\right) .
\end{array}
$$

Then $f$ is continuous since the definitions agree at the places where 
they overlap, namely, the values $\theta_{1}, \theta_{2}$ and $2 \pi$ or 0 for $\theta$. Also since $f_{1}$ and $f_{2}$ are locally simple and the image set under $f$ of the arc given by $\pi / 2 \leqq \theta \leqq \theta_{1}$ has just $w$ in common with $C_{2}$ whereas $f(z) \in C_{2}$ for $\theta_{1} \leqq \theta \leqq 3 \pi / 2$ and a similar situation holds in the neighborhood of $\theta_{2}$, it follows that $f$ is locally simple. Finally, since $f$ agrees with $f_{1}$ over an interval of $\theta$ of length greater than $\pi / 2$ and also with $f_{2}$ over a similar interval, it follows that $f$ maps the circle $|z|=1$ onto $C_{1}+C_{2}$.

(3.11) Corollary. Any continuum which is the sum of a finite number of continua each of which has a locally simple representation on the circle itself has a locally simple representation on the circle.

(3.2) THEOREM. In order that a continuum $M$ have a locally simple representation on the circle it is necessary and sufficient that it be the sum of a finite number of doubly extensible arcs.

For suppose the mapping $f(C)=M$ is locally simple, where $C$ is a circle. Then for some $e>0$, every arc of $C$ of diameter less than $3 e$ maps topologically onto its image in $M$. Thus if we subdivide $C$ into a finite number of arcs of diameter less than $e$, the image of each of these arcs is a doubly extensible simple arc in $M$ and the sum of these images is $M$ itself.

On the other hand, suppose $M$ is the sum of a finite number of double extensible $\operatorname{arcs} \alpha_{1}, \alpha_{2}, \cdots, \alpha_{n}$. Since then no point of $M$ can be an end point, by (2.11) each $\alpha_{i}$ is contained in either a simple closed curve, a $\theta$-curve, an 8 -curve, or a dumbbell curve in $M$. Thus each $\alpha_{i}$ is contained in either a dumbbell curve or in the sum of at most two simple closed curves in $M$. Accordingly, we can write $M=\sum_{1}^{n} \alpha_{i}=\sum_{1}^{m} C_{i}$, where each $C_{i}$ is either a simple closed curve or a dumbell curve. Since clearly each $C_{i}$ has a locally simple representation on the circle, it follows by (3.11) that so also has $M$.

(3.3) THEOREM. In order that a continuum have a locally simple representation on the circle it is necessary and sufficient that it be the sum of a finite number of simple closed curves and dumbbell curves.

For if $M$ satisfies this condition, it has a locally simple representation on the circle by (3.11), since each of the component curves has such a representation; and if $M$ has such a representation, by (3.2) and its proof, $M$ is the sum of a finite number of curves of the required type.

(3.31) A connected graph has a locally simple representation on the circle if and only if it has no end points.

(3.4) Theorem. A cyclic continuum $M$ has a locally simple repre- 
sentation on the circle if and only if it is the sum of a finite number of simple closed curves.

For if $M$ is such a sum, it has a locally simple representation on the circle by (3.11). On the other hand, if $M$ has this type of representation, by (3.2) it is the sum of a finite number of doubly extensible arcs. Then since each such arc lies in a cyclic graph in $M$ by (2.2), $M$ is the sum of an equal number of cyclic graphs. But each cyclic graph is the sum of a finite number of simple closed curves, because each edge lies on a simple closed curve in the graph. Accordingly, $M$ is the sum of a finite number of simple closed curves.

Example. It is now clear that the continuum described at the end of $\$ 2$ admits no locally simple representation on the circle, since it is not a finite sum of simple close curves or of extensible arcs.

4. Representations of boundary curves. A boundary curve is a locally connected continuum every true cyclic element of which is a a simple closed curve, or equivalently, a locally connected continuum which is homeomorphic with the boundary of a plane region. For this and other terms used in $\$ \S 4$ and 5 , the reader is referred to the author's book [2].

(4.1) THEOREM. The following properties are equivalent for locally connected continua $M$ :

(i) $M$ has only a finite number of nodes and no end points;

(ii) there exists a $d>0$ such that each node of $M$ is of diameter not less than $d$;

(iii) there exists an $e>0$ such that each component of the complement in $M$ of an arbitrary point of $M$ is of diameter not less than $e$.

First, (i) implies (ii), since every degenerate node is an end point. To show that (ii) implies (iii), we have only to take $e=d$. Then since the closure of any component of the complement in $M$ of a point of $M$ contains at least one node of $M$, (iii) is satisfied. Finally, if (iii) holds, $M$ can have neither an end point nor an infinity of nodes, because in either case we could find a cut point $p$ of $M$ and a component of $M-p$ of diameter less than any given positive number since the nondegenerate nodes can form a null sequence at most.

(4.2) THEOREM. If a continuum $M$ has a locally simple representation on the circle, it has the properties listed in (4.1).

For by (3.3), $M=\sum_{1}^{n} C_{i}+\sum_{1}^{m} D_{j}$, where each $C_{i}$ is a simple closed curve and each $D_{j}$ is a dumbbell curve. Let $C_{1 j}$ and $C_{2 j}$ be the two simple closed curves in $D_{j}$ for each $j \leqq m$, and set $e=\min \left[\delta\left(C_{i}\right)\right.$, 
$\left.\delta\left(C_{1 j}\right), \delta\left(C_{2 j}\right)\right]$ for all $i \leqq n$ and $j \leqq m$. Then for any $p \in M$ and any component $R$ of $M-p$, we must have $\delta(R) \geqq e$, because $\bar{R}$ contains either some $C_{i}$, some $C_{1 j}$, or some $C_{2 j}$. Thus (iii) holds.

(4.3) TheOREM. Let $B$ be any boundary curve having one of the properties in (4.1). Then any light non-alternating mapping $f(C)=B$ of a circle $C$ onto $B$ is locally simple.

For by property (iii), there exists an $e>0$ such that for any $p \in B$, each component of $B-p$ is of diameter not less than $e$. Let $\delta>0$ be chosen so that the image under $f$ of any subset of $C$ of diameter less than $\delta$ is of diameter less than $e$. Then on any arc of $C$ of diameter less than $\delta, f$ must be 1-1. For otherwise there would exist a point $p$ of $B$ and an arc $x y$ of $C$ with $\delta(x y)<\delta$ and $f(x)=f(y)=p, f(x y-x-y)$ $C B-p$. But this is impossible, because $f(x y-x-y)$ is a component of $B-p$ since $f$ is non-alternating, and $\delta[f(x y)]<e$. Accordingly, $f$ is locally simple.

(4.4) Theorem. A boundary curve has a locally simple representation on the circle if and only if it has the properties in (4.1).

This is a direct consequence of (4.2), (4.3), and the fact that any boundary curve has light non-alternating representations on the circle.

5. Cyclic connectedness. We conclude by showing how the imbedding result (2.2) affords a simple approach to the cyclic connectedness theorem. First we give two additional elementary results.

(5.1) Any two points of a locally connected continuum $M$ which are interior points of arcs of $M$ (thus actually any two non-end points) lie together on a doubly extensible arc in $M$.

For let $a$ and $b$ be two such points and let $\alpha$ and $\beta$ be disjoint arcs in $M$ having $a$ and $b$ respectively as interior points. Let $a_{1} b_{1}$ be any arc in $M$ with $a_{1} b_{1} \cdot \alpha=a_{1}, a_{1} b_{1} \cdot \beta=b_{1}$. Then clearly the arc $a b=a_{1} b_{1}$ $+a_{1} a$ (of $\left.\alpha\right)+b_{1} b$ (of $\beta$ ) is doubly extensible in $M$.

(5.2) Any two points $a$ and $b$ of a cyclic graph $G$ lie together on $a$ simple closed curve in $G$.

For let $S$ be the set of all points $x$ in $G$ such that some simple closed curve $C_{x}$ in $G$ contains both $a$ and $x$. Then $S$ i, closed. For if $S$ contains a point $x$ of an open edge $E$ of $G$, the simple closed curve $C_{x}$ must contain $\bar{E}$ so that $S \supset \bar{E}$. However, $S$ is also open in $G$. For as just seen, $S$ contains every open edge of $G$ which it intersects; and if a vertex $x$ of $G$ is in $S$ and $C_{x}$ is a simple closed curve in $G$ containing $a+x$ and $E$ is an open edge of $G$ having $x$ as an end point but not 
intersecting $C_{x}, E$ lies in an arc $x y$ of $G$ with $x y \cdot C_{x}=x+y$. Since then $x y+\operatorname{arc} x a y$ of $C_{x}$ is a simple closed curve containing $E$, we have $E \subset S$ and thus $x$ is interior to $S$. Hence $S$ is both open and closed in $G$ and accordingly $S=G$.

Clearly (5.1) and (5.2) together with (2.2) give:

(5.3) Any two points of a cyclic locally connected continuum $C$ which are interior point of arcs of Clie together on a simple closed curve in $C$.

Now if we make use of the fact, somewhat harder to prove, that every point of a cyclic locally connected continuum $C$ is interior to some arc in $C$, we can omit the clause "which are interior points of arcs of $C$ " from (5.3) and we have the cyclic connectedness theorem.

\section{BIBLIOGRAPHY}

1. M. Morse and M. Heins, Topological methods in the theory of functions of a single complex variable. I. Deformation types of locally simple plane curves, Ann. of Math. vol. 46 (1945) pp. 600-624.

2. G. T. Whyburn, Analytic topology, Amer. Math. Soc. Colloquium Publications, vol. 28, New York, 1942.

UNIVERSITY OF VIRGINIA 